Bull. Austral. Math. Soc.

Vol. 45 (1992) [157-162]

\title{
ON THE PASS-EQUIVALENCE OF LINKS
}

\author{
YAN-LOI WONG
}

We give a simple geometric proof that the Jones polynomial at the value $i$ of an oriented link is invariant under pass-equivalence.

\section{INTRODUCTION}

The Arf invariant of a knot or more generally a proper oriented link was introduced in [6]. It was shown by Murakami in [5] that the value $V_{L}(i)$ of the Jones polynomial at $i$ is a suitable generalisation of the Arf invariant for an arbitrary oriented link $L$. In fact Murakami computed that $V_{L}(i)$ equals $(-\sqrt{2})^{c(L)-1}(-1)^{\operatorname{Arf}(L)}$ if $L$ is a proper oriented link and equals zero if $L$ is not proper, where $c(L)$ denotes the number of components of $L$. On the other hand, Kauffman introduced in [2] the concept of pass-equivalence or equivalently $\Gamma$-equivalence of links. It was shown in [2] that any oriented link is pass-equivalent to either the unlink, the unlink disjoint union a trefoil or the unlink disjoint union a connected sum of Hopf links. This together with Murakami's result implies the following:

THEOREM. Two oriented links $L$ and $L^{\prime}$ are pass-equivalent if and only if $V_{L}(i)=$ $V_{L^{\prime}}(i), c(L)=c\left(L^{\prime}\right)$ and $n(L)=n\left(L^{\prime}\right)$, where $n(L)$ is the number of components $K$ of $L$ such that $l k(K, L-K)$ is odd.

In this paper we shall give a direct geometric proof of the fact that $V_{L}(i)$ is invariant under pass-equivalence and hence the above result and Murakami's result. All the links considered are oriented.

\section{Pass-equivalence and T-equivalence}

A pass-move on a link diagram is a move of one of the following two forms:
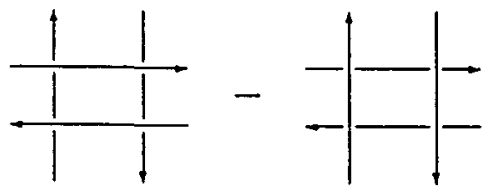

or

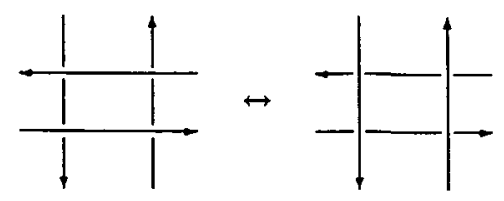

Received 27 February 1991

The author would like to thank Professor Murakami for pointing out an error in an earlier version of this paper.

Copyright Clearance Centre, Inc. Serial-fee code: 0004-9729/92 \$A2.00+0.00. 


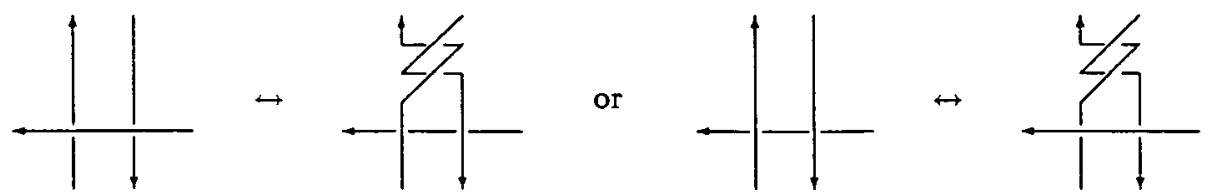

A $\Gamma$-move on a link diagram is a move of one of the above two forms:

Definition 1.1: Two links are pass-equivalent ( $\Gamma$-equivalence) if one can be obtained from the other by a finite combination of pass-moves ( $\Gamma$-moves) and ambient isotopies.

We shall use $\sim$ to denote pass-equivalence and $\equiv$ to denote the equivalence of being ambient isotopic. Next we recall the following two results from [2] and [3].

Proposition 1.2. Two links are pass-equivalent if and only if they are $\Gamma$ equivalent.

Proposition 1.3. Any link is pass-equivalent to one of the following three forms:

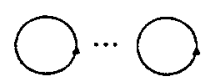

(a)

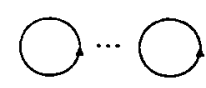

(b)

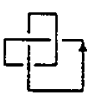

b)
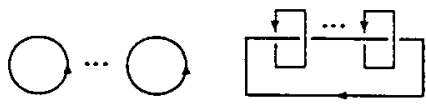

(c)

where in (a) the Arf invariant is 0 , in (b) the Arf invariant is 1 and in (c) the number of components minus the number of unknots is even.

\section{ThE INVARIANT $V_{L}(i)$ OF A LINK $L$}

$V_{L}(i)$ is the value of the Jones polynomial of $L$ at $i$. It satisfies the following two axioms:

(i) $V_{\text {unknot }}(i)=1$;

(ii) $V_{L_{+}}(i)+V_{L_{-}}(i)=-\sqrt{2} V_{L_{0}}(i)$, where $L_{+}, L_{0}$ are three links identical except within a ball where they have a projection as follows:
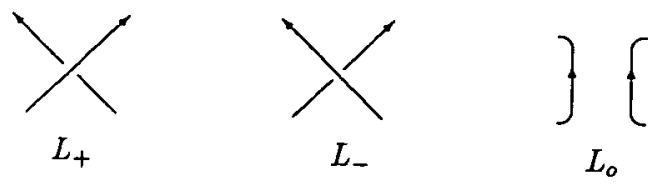

In fact (i) and (ii) uniquely determine the numerical invariant $V(i)$.

For any two links $L_{1}$ and $L_{2}, l k\left(L_{1}, L_{2}\right)$ denotes the total linking number of $L_{1}$ and $L_{2}$. We say that a link $L$ is proper if $l k(K, L-K)$ is even for every component 
$K$ in $L$, otherwise it is said to be non-proper. For example the link in Proposition 1.2 (c) is non-proper. Next we recall the following result in [1].

JONES REVERSING RESULT: If $L^{\prime}$ is obtained from $L$ by reversing the orientation of one component that has linking number $m$ with the remaining components of $L$, then $V_{L^{\prime}}(t)=t^{-3 m} V_{L}(t)$.

Suppose $L$ is a non-proper link. Let $K$ be a component of $L$ such that $l k(K, L-K)=m$ is odd. Then by reversing the orientation of $K$, we have $V_{L^{\prime}}(i)=i^{-3 m} V_{L}(i)= \pm i V_{L}(i)$. From axiom (ii), $V_{L}(i)$ is always a real number. Hence $V_{L}(i)=0$.

To prove the main theorem we need two lemmas which are also use in [4] to prove Murakami's result.

Lemma 2.1. Let $L$ be an oriented link and $L^{\prime}$ the link constructed by banding together two distinct components of $L$. If $L$ is proper, then $L^{\prime}$ is proper and $V_{L^{\prime}}(i)=$ $-\sqrt{1 / 2} V_{L}(i)$.

Proof: A calculation of linking numbers shows that if $L$ is proper, then $L^{\prime}$ is proper. By cutting the band within a 3 -ball surrounding the band, we have the following skein triple.
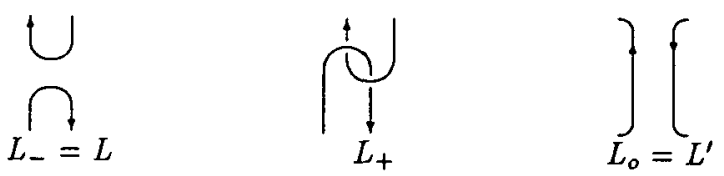

Since $L_{-}$is non-proper, $V_{L_{-}}(i)=0$. Hence $V_{L^{\prime}}(i)=-\sqrt{1 / 2} V_{L}(i)$.

Similarly one can prove the next lemma.

LEMмA 2.2. Let $L, L^{\prime}$ and $L^{\prime \prime}$ be three oriented links identical except within a ball where they have a projection as shown below:
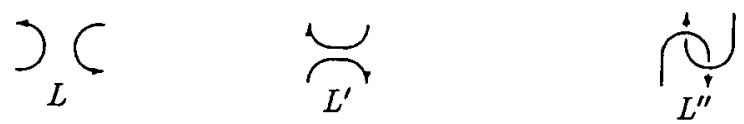

where the two strings in $L$ belong to the same component. Suppose $L$ is proper. Then precisely one of $L^{\prime}$ and $L^{\prime \prime}$ is proper. Furthermore if $L^{*} \in\left\{L^{\prime}, L^{\prime \prime}\right\}$ is proper, then $V_{L^{*}}(i)=-\sqrt{2} V_{L}(i)$.

LEMMA 2.3. $V_{L \#} T_{\text {refoil }}(i)=-V_{L}(i)$. 
Proof: $V_{L \# \text { Trefoil }}(i)=V_{\text {Trefoil }}(i) V_{L}(i)=-V_{L}(i)$.

\section{Proof of THE ThEOREM}

Proposition 3.1. If $L$ and $L^{\prime}$ are $\Gamma$-equivalent, then $V_{L}(i)=V_{L^{\prime}}(i)$.

Proof: It suffices to show that $V(i)$ does not change with the two $\Gamma$-moves. For this, we shall show that if $L$ and $L^{\prime}$ are links identical except within a ball where they have a projection as shown below,
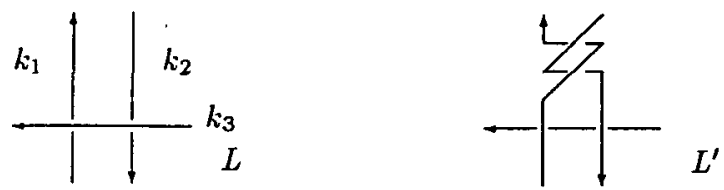

then $V_{L}(i)=V_{L^{\prime}}(i)$. Notice that $\Gamma$-moves or pass-moves preserve properness. Therefore in the case of non-proper links, $V_{L}(i)=V_{L^{\prime}}(i)$. Hence we only need to consider proper links. There are three cases.

CASE 1. $\left(k_{1}, k_{2}\right.$ and $k_{3}$ belong to the same components of $L$.) We can represent a $\Gamma$-move by a sequence of taking connected sums of the trefoils or the components of $L$. By keeping track of the value $V_{L}(i)$, we will show that $V_{L}(i)=V_{L^{\prime}}(i)$. This is shown as follows.

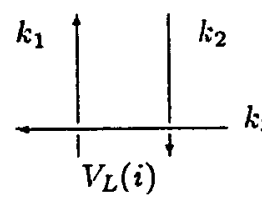

lemma 2.3

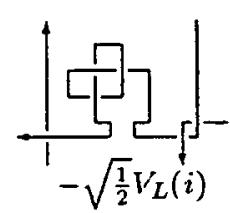

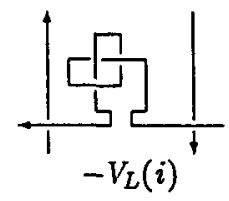

lemma 2.1

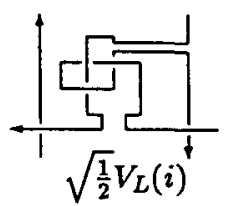

lemma 2.1

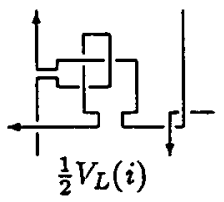

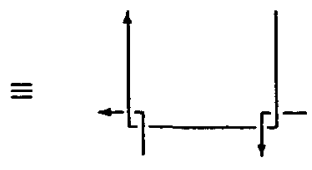

Here the three strings in the last diagram belong to a single component. By taking a connected sum or a connected sum with a twist of the two lower strings, we get two possibilities:

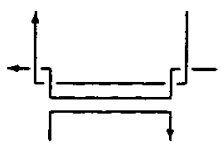

or

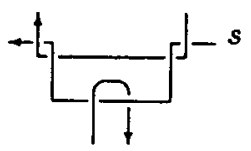


But in the second case the linking number of $s$ and the rest of the other components is odd so that it is not proper. By Lemma 2.2 the first link is proper and its value of $V(i)$ is equal to $-\sqrt{1 / 2} V_{L}(i)$. That is

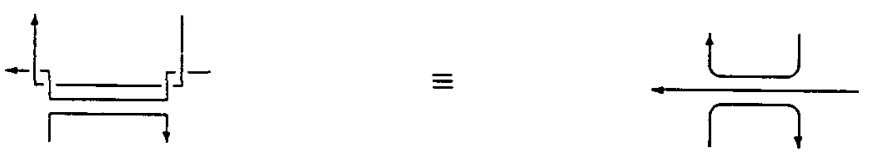

and its value of $V_{L}(i)$ equals $-\sqrt{1 / 2} V_{L}(i)$.

Again we take a connected sum or a connected sum with a twist of the upper and lower strings. We then have the cases:

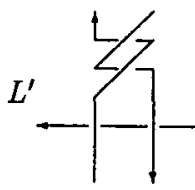

or

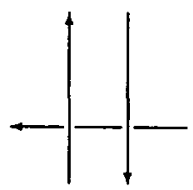

Since the latter case gives a non-proper link, we perform the operation of taking a connected sum with a twist to get $L^{\prime}$. By Lemma $2.2 V_{L^{\prime}}(i)=V_{L}(i)$.

CASE 2. (Only two of the strings belong to the same component.) By taking a connected sum or a connected sum with a twist of the two strings, say $k_{1}$ and $k_{2}$ of the same component outside the ball, we get two links. By Lemma 2.2 precisely one of them is proper and for that one $L^{*}, V_{L^{*}}(i)$ equals $-\sqrt{2} V_{L}(i)$. Inside the ball we still have the same link diagram but the three strings now belong to different components of $L^{*}$. Hence we can apply the result of Case 1 to conclude that if $L^{* *}$ is the link obtained by performing a $\Gamma$-move on $L^{*}$ within the ball, then $V_{L^{* *}}(i)=V_{L^{*}}(i)=-\sqrt{2} V_{L}(i)$. Now we take a connected sum of the knots $k_{1}$ and $k_{2}$. We get a proper link which is $L^{\prime}$ and by Lemma $2.1, V_{L^{\prime}}(i)=-\sqrt{1 / 2} V_{L^{* *}}(i)=V_{L}(i)$.

CASE 3. (All three strings belong to the same component.) We can apply the same argument as in Case 2 to two of the strings and reduce this case to Case 2. This completes the proof.

Proof of The theorem: $(\Rightarrow)$ That $V_{L}(i)=V_{L^{\prime}}(i)$ is proved in Proposition 3.1. Since pass-equivalence does not change the number of components $K$ such that $l k(K, L-K)$ is odd, we have $n(L)=n\left(L^{\prime}\right)$. Obviously $c(L)=c\left(L^{\prime}\right)$.

$(\Leftarrow)$ By Proposition 1.3, any link is pass-equivalent to one of the form (a), (b) and (c) as shown in Proposition 1.3. If both $L$ and $L^{\prime}$ are not proper, then they are pass-equivalent to a link of the form (c). Since $c(L)=c\left(L^{\prime}\right)$ and $n(L)=n\left(L^{\prime}\right)$, we 
must have $L \sim L^{\prime}$. If $L$ and $L^{\prime}$ are both proper, then they are pass-equivalent to a link of the form (a) and (b). By Proposition 3.1, we have

$$
V_{L}(i)= \begin{cases}(-\sqrt{2})^{c(L)-1} & \text { if } L \sim(a) \\ -(-\sqrt{2})^{c(L)-1} & \text { if } L \sim(b)\end{cases}
$$

Since $V_{L}(i)=V_{L^{\prime}}(i)$, we must have $L$ and $L^{\prime}$ both pass-equivalent to either the form (a) or (b). Hence $L \sim L^{\prime}$. This completes the proof of the theorem.

Corollary. (Murakami [5]) For any oriented link $L$,

$$
V_{L}(i)= \begin{cases}(-\sqrt{2})^{c(L)-1}(-1)^{A r f(L)} & \text { if } L \text { is proper } \\ 0 & \text { if } L \text { is non-proper. }\end{cases}
$$

\section{REFERENCES}

[1] V. Jones, 'A polynomial invariant for knots via von Neumann algebras', Bull. Amer. Math. Soc. 12 (1985), 103-111.

[2] L. Kauffman, 'Link manifolds', Michigan Math. J. 21 (1974), 33-44.

[3] L. Kauffman, 'The Arf invariant of classical knots', in Contemp. Math. 44: Combinatorial Methods in Topology and Algebraic Geometry, pp. 101-116.

[4] W. Lickorish and K. Millett, 'Some evaluations of link polynomials', Comment. Math. Helv. 61 (1986), 335-338.

[5] H. Murakami, 'A recursive calculation of the Arf invariant of a link', J. Math. Soc. Japan 38 (1986), 335-338.

[6] R. Robertello, 'An invariant of knot cobordism', Comm. Pure Appl. Math. XVIII (1965), 543-555.

Department of Mathematics

National University of Singapore

Singapore 0511

Republic of Singapore 